\title{
Heterogeneity of Plasma von Willebrand Factor Multimers Resulting from Proteolysis of the Constituent Subunit
}

\author{
Judith A. Dent, Miriam Galbusera, and Zaverio M. Ruggeri \\ Roon Research Center for Arteriosclerosis and Thrombosis, Division of Experimental Hemostasis and Thrombosis \\ of the Department of Molecular and Experimental Medicine, and Committee on Vascular Biology, \\ Scripps Clinic and Research Foundation, La Jolla, California 92037
}

\begin{abstract}
In this report we demonstrate that proteolytic cleavage of the constituent subunit is one of the causes determining the heterogeneous size distribution of plasma von Willebrand factor (vWf) multimers. As shown by two-dimensional nonreduced/ reduced agarose/polyacrylamide gel electrophoresis, the structure of circulating vWf molecules may deviate from that represented by assemblage of a variable number of identical subunits. Indeed, even though the largest multimers in normal plasma appear to be composed predominantly of intact 225-kD subunits, those of intermediate and smaller size contain also 189-, 176-, and 140-kD proteolytic fragments. Different subunit composition patterns are repeated regularly in multimers of increasing molecular mass, yielding series of bands with similar structure. One of these series consists of molecules without evidence of proteolytic fragmentation, and its smallest member appears to be a dimer of $225-\mathbf{k D}$ subunits. Type IIA von Willebrand disease, characterized by absence of the largest multimers, displays a pattern wherein the fragments of 176 and 140 $\mathrm{kD}$ are relatively increased, that of $189 \mathrm{kD}$ is markedly decreased or absent, but the composition of individual multimers is otherwise similar to that of species seen also in normal plasma. In contrast to those in the circulation, all normal platelet $\mathrm{vWf}$ multimers contain only intact subunit. These results suggest that proteolytic cleavage of plasma vWf subunits occurs after release from cellular sites, whereas platelet $v W f$ stored in $\alpha$-granules is protected from proteolysis. These findings provide information that may be relevant for understanding the normal processing of $\mathbf{v W f}$ multimers and for elucidating the pathogenesis of some of the congenital and acquired structural abnormalities of this molecule. (J. Clin. Invest. 1991. 88:774-782.) Key words: platelets $\bullet$ adhesive protein • satellite band $\bullet$ two-dimensional gel electrophoresis
\end{abstract}

\section{Introduction}

The adhesive protein von Willebrand factor (vWf $)^{1}$ exists in plasma as a series of heterogeneous multimers ranging in size

Address correspondence and reprint requests to Dr. Zaverio M. Ruggeri, BCR-8, Research Institute of Scripps Clinic, 10666 North Torrey Pines Road, La Jolla, CA 92037.

Received for publication 31 May 1990 and in revised form 21 March 1991.

1. Abbreviations used in this paper: HGT, LGT, high, low gelling temperature; KIU, kallikrein inhibitory units; NEM, $N$-ethylmaleimide; vWf, von Willebrand factor.

J. Clin. Invest.

(c) The American Society for Clinical Investigation, Inc. 0021-9738/91/09/0774/09 \$2.00

Volume 88, September 1991, 774-782 from $\sim 450 \mathrm{kD}$ to in excess of $10,000 \mathrm{kD}(1)$. The primary translation product (pre-pro-vWf) is a polypeptide of 2813 amino acids with a calculated molecular mass of $307 \mathrm{kD}(2)$, not including the $\sim 15-19 \%$ carbohydrate. After removal of the signal peptide, the resulting pro-vWf subunits $(3,4)$ are engaged in a complex biosynthetic process thought to begin with the formation of dimers through disulfide bond linkage at the carboxyl terminal ends; then, these protomeric units of the multimeric series are assembled into higher order multimers by disulfide bonding at amino terminal ends $(5,6)$. A propeptide of 741 amino acids is cleaved from the amino terminus of each prosubunit at the time of multimerization $(3,4)$, resulting in formation of the mature vWf subunit. This has a calculated polypeptide molecular mass of $226 \mathrm{kD}$ (7); including the 22 oligosaccharide chains, the subunit molecular mass is $\sim 270$ $\mathrm{kD}$, whereas it is estimated at $225 \mathrm{kD}$ from relative electrophoretic mobility in polyacrylamide gels containing SDS. The released propeptide, with an estimated molecular mass of 100 $\mathrm{kD}$, circulates as a plasma protein of still undetermined function known as von Willebrand antigen II $(8,9)$.

Analysis of nonreduced plasma vWf by means of SDS-agarose gel electrophoresis in a discontinuous buffer system demonstrates a complex pattern of repeating multimers, characterized by the presence of a more prominent band surrounded by three (10) or more (11) satellite bands, depending on the resolving power of the gel system used. Because these bands are unevenly spaced and differ considerably in relative intensity, they do not fit the expected pattern of a series of multimers constituted by a regularly increasing number of identical subunits. Certain of these bands may be missing or migrate with an aberrant mobility in von Willebrand disease variants (10-17), suggesting that the complex multimeric structure of plasma $\mathrm{vWf}$ may have pathophysiological significance. Indeed, proteolytic fragments of the vWf subunit are present in normal individuals, increased in type IIA and IIB von Willebrand disease, and decreased or absent in variants with an aberrant multimeric structure (Types IIC, IID, and IIE) (14). Mapping with anti-vWf monoclonal antibodies has localized the origin of the 176- and 140-kD fragments at the carboxyl and amino terminal ends of the subunit, respectively, with the minor $189-\mathrm{kD}$ fragment apparently overlapping both of them (18). More recently, work from our laboratory has led to the identification of the peptide bond, $\mathrm{Tyr}^{842}-\mathrm{Met}^{843}$ in the mature subunit, that is cleaved to generate the 140 - and $176-\mathrm{kD}$ subunit species (19). Whether and how these fragments are represented in vWf multimers has not been clarified to date.

In this study, we have used a two-dimensional nonreduced/ reduced agarose/polyacrylamide gel system to demonstrate that the multiplicity of bands resolved from plasma vWf is due, in part, to variable content of different proteolytic fragments of the $225-\mathrm{kD}$ subunit. In contrast, $\mathrm{vWf}$ stored within platelets is composed exclusively of unproteolyzed mature subunit. Thus, cleavage of the constituent subunit may be one of the mecha- 
nisms by which vWf is processed physiologically at the time of, or after secretion into the circulation. This may have consequences for the function of the molecule and may help define structural abnormalities in congenital and acquired alterations of vWf.

\section{Methods}

All human subjects described in this report gave their informed consent for participation in the study, according to the declaration of Helsinki. The patients with Type IIA von Willebrand disease fulfilled all the criteria for this diagnosis, including prolonged bleeding time, markedly decreased ristocetin cofactor activity, and lack of large and intermediate vWf multimers in plasma. Normal individuals and patients denied consuming any drugs in the week preceding the study.

Reagents. Low gelling temperature (LGT) agarose (Type VII), aprotinin and $N$-ethylmaleimide (NEM) were from Sigma Chemical Co., St. Louis, MO. Leupeptin was from Chemicon, Los Angeles, CA. High gelling temperature agarose (HGT[P]) and Gel bond were from FMC Corp., Rockland, ME. Cyanogen bromide-activated Sepharose was from Pharmacia Fine Chemicals, Piscataway, NJ. Electrophoresispure reagents were obtained from Bio-Rad, Richmond, CA. Rabbit anti-mouse IgG was from Zymed, South San Francisco, CA. All other reagents were of the highest grade available.

Preparation of plasma and platelet samples. Blood was drawn from an antecubital vein through a 19-gauge needle into a polypropylene syringe, and immediately transferred into polypropylene tubes containing either $1 / 6$ vol of acid-citrate-dextrose anticoagulant, $\mathrm{pH} 4.5$ (for platelet preparation), or $1 / 10 \mathrm{vol}$ of $0.11 \mathrm{M}$ trisodium citrate (for plasma preparation) with 200 kallikrein inhibitory units (KIU) aprotinin per ml, $1 \mathrm{mM}$ leupeptin, $5 \mathrm{mM}$ EDTA, and $6 \mathrm{mM}$ NEM (final concentrations in plasma, calculated assuming an average plasma volume of $0.6 \mathrm{ml}$ per $1 \mathrm{ml}$ of blood). Platelet-poor plasma was prepared from citrated blood by centrifugation at $3,000 \mathrm{~g}$ for $20 \mathrm{~min}$ at room temperature $\left(22-25^{\circ} \mathrm{C}\right)$. Plasma was rapidly separated from sedimented cells and stored at $-70^{\circ} \mathrm{C}$ until tested. Platelet-rich plasma was pooled from three consecutive centrifugations performed at room temperature at $1,100 \mathrm{~g}$ for $75 \mathrm{~s}$ each. Platelets were washed by pelleting them twice at $1,100 \mathrm{~g}$ for $20 \mathrm{~min}$ at room temperature and resuspending each time in a solution containing $0.015 \mathrm{M}$ sodium citrate, $0.028 \mathrm{M}$ dextrose, $0.125 \mathrm{M}$ sodium chloride, and $0.005 \mathrm{M}$ EDTA, brought to pH 6.5 with $1 \mathrm{M}$ citric acid (buffer A). After a third centrifugation, the pellet was resuspended in a $\mathrm{pH} 7.4$ solution, containing $0.14 \mathrm{M} \mathrm{NaCl}$, $0.01 \mathrm{M}$ Tris-HCl, and $0.005 \mathrm{M}$ EDTA (buffer B), in a volume corresponding to one-tenth of the initial blood volume.

Immunoisolation of plasma $v W f$. Citrated plasma $(5-10 \mathrm{ml})$ was used for immunoisolation of $\mathrm{vWf}$, performed as described previously (14), in the presence of $1 \mathrm{mM}$ leupeptin, $5 \mathrm{mM}$ EDTA, $6 \mathrm{mM} \mathrm{NEM}$, and $200 \mathrm{KIU}$ aprotinin per $\mathrm{ml}$. In brief, a monoclonal anti-vWf antibody (2.2.9), prepared and characterized as described previously $(20$, 21 ), was coupled to cyanogen bromide-activated Sepharose CL-4B at a ratio of $2.5 \mathrm{mg}$ of antibody per milliliter of beads. IgG for the coupling procedure was purified from ascitic fluid by affinity chromatography on a Protein A column (Sigma Chemical Co.), as previously described (22). $1 \mathrm{ml}$ of beads had the ability to bind $\sim 15 \mu \mathrm{g}$ of vWf. The antibody 2.2.9 recognizes an epitope located in the carboxyl terminal region of the mature vWf subunit, within residues 1366 and 2050 (unpublished results). After incubation with plasma and washing of the beads (14), vWf was eluted with $2 \%$ SDS and used for electrophoretic analysis and immunoblotting. Samples were concentrated to a final volume of 40-60 $\mu \mathrm{l}$ using Amicon Centricon 30 microconcentrators (Amicon Corp., Danvers, MA).

Immunoisolation of platelet $v W f$. Platelet $\mathrm{vWf}$ was prepared by sonication of $1 \mathrm{ml}$ of washed platelet suspension. Platelets were disrupted on ice in buffer $B$ either in the presence of the four proteinase inhibitors, $10 \mathrm{mM}$ EDTA, $6 \mathrm{mM}$ NEM, $1 \mathrm{mM}$ leupeptin, and $200 \mathrm{KIU}$ aprotinin per $\mathrm{ml}$, or $10 \mathrm{mM}$ EDTA only, or no inhibitors. A sonicator cell disrupter (model W.220-F; Heat Systems-Ultrasonics, Inc., Plainview, NY) was used, giving four 5-s bursts punctuated with 10-s rest periods with an output control set at $\sim 40$. Cellular debris was removed by centrifugation at $20,000 \mathrm{~g}$ for $30 \mathrm{~min}$ at $5^{\circ} \mathrm{C}$, and platelet vWf was immunopurified using the same procedure described above for plasma vWf.

Two-dimensional SDS gel electrophoresis. Discontinuous SDS agarose gel electrophoresis has been described in detail previously $(10,11)$. Briefly, two types of gels were cast for resolution in the first dimension: "short", intermediate resolution gels; and "long", high resolution gels. For the first type, resolving gels $(22 \mathrm{~cm}$ wide $\times 9 \mathrm{~cm}$ long $\times 1.0 \mathrm{~mm}$ thick) of $1.4 \%$ LGT agarose were cast on Gel-bond film between glass plates and then allowed to polymerize at $4^{\circ} \mathrm{C}$ for $3 \mathrm{~h}$ before pouring the $0.8 \%$ HGT(P) agarose stacking gel $(2.5 \mathrm{~cm}$ long). 10 wells, $10 \mathrm{~mm} \times 2$ $\mathrm{mm}$, were cut $1 \mathrm{~cm}$ from the stacking gel/resolving gel interface, and 20 $\mu \mathrm{l}$ of concentrated immunopurified $\mathrm{vWf}$ or plasma (1:20 dilution) was applied after addition of tracking dye. Electrophoresis was performed horizontally at a constant current of $0.41 \mathrm{~mA}$ per $\mathrm{cm}$ in an LKB Multiphor apparatus (LKB, Pleasant Hill, CA) at $16^{\circ} \mathrm{C}$ for $18 \mathrm{~h}$ or until the tracking dye was $1.0 \mathrm{~mm}$ from the anodal wick. For the second type, resolving gels $(9 \mathrm{~cm}$ wide $\times 22 \mathrm{~cm}$ long $\times 1.0 \mathrm{~mm}$ thick) were cast with $2.35 \%$ LGT agarose. Each sample was prepared and processed as above, and applied into one of four sample wells. Horizontal electrophoresis was performed in an LKB Multiphor apparatus at $0.75 \mathrm{~mA}$ per $\mathrm{cm}$ across the gel at $16^{\circ} \mathrm{C}$ for $18 \mathrm{~h}$ or until the tracking dye was 1.0 $\mathrm{mm}$ from the anodal wick. Purification of the proteins used as markers of molecular mass was performed as previously described for mouse IgG (22), IgM (23), human fibrinogen (24), and fibronectin (25).

For resolution in the second dimension, 5\% SDS-polyacrylamide gels were prepared as described by Laemmli (26), with stacking and running gel. First dimension agarose gels were washed in deionized water for $60 \mathrm{~min}$ after electrophoresis. Individual lanes were excised

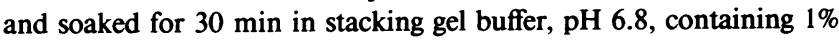
SDS and $65 \mathrm{mM}$ DTT. For comparison, adjacent lanes from the agarose gels were fixed and processed as previously detailed for visualization with ${ }^{125} \mathrm{I}$-labeled rabbit anti-human vWf and subsequent autoradiography (10). Lanes from short gels were applied as single strips and sealed to the top of the polyacrylamide stacking gel. Long gels were bisected and applied to two separate second dimension gels. The lanes were cemented in place with $0.8 \%$ HGT $(\mathrm{P})$ agarose, $1 \%$ SDS, pH 6.8. A 2-mm layer of agarose was poured above the strip to provide a level, horizontal surface, and $750 \mu \mathrm{l}$ of $65 \mathrm{mM}$ DTT, $2 \%$ SDS, $10 \%$ glycerol in sample buffer, $\mathrm{pH} 7.0$, applied on top of this. Electrophoresis was performed vertically at $22-25^{\circ} \mathrm{C}$, with $25 \mathrm{~mA}$ applied during the run in the stacking gel and $35 \mathrm{~mA}$ in the running gel.

Immunoblotting. Polypeptides resolved by SDS-PAGE were transferred onto nitrocellulose membranes in $25 \mathrm{mM}$ Tris, $192 \mathrm{mM}$ glycine, $20 \%\left(\mathrm{vol} / \mathrm{vol}\right.$ ) methanol buffer at $0.25 \mathrm{~A}$ for $18 \mathrm{~h}$ at $3^{\circ} \mathrm{C}$, and reacted with a pool of 80 anti-vWf monoclonal antibodies, followed by ${ }^{125}$ I-labeled rabbit anti-mouse IgG as described previously (14). These monoclonal antibodies were prepared and characterized as reported previously $(18,20,21)$. For each sample processed for two-dimensional analysis, an aliquot of starting material was reduced in $65 \mathrm{mM}$ DTT and applied directly to the SDS-polyacrylamide gel in a lane at the far left to serve as a standard (one-dimensional analysis).

\section{Results}

Electrophoresis of nonreduced normal plasma vWf in intermediate resolution SDS-agarose gels resulted in the separation of a number of apparent "triplets" each characterized by a central band, predominant in intensity, and two fainter satellite bands, one above and the other below it (Fig. $1 A$ ). This previously described pattern (10) was clearly visible in small and intermediate multimers but not in the larger ones, because resolution decreased with increasing multimer size. Marker proteins rang- 

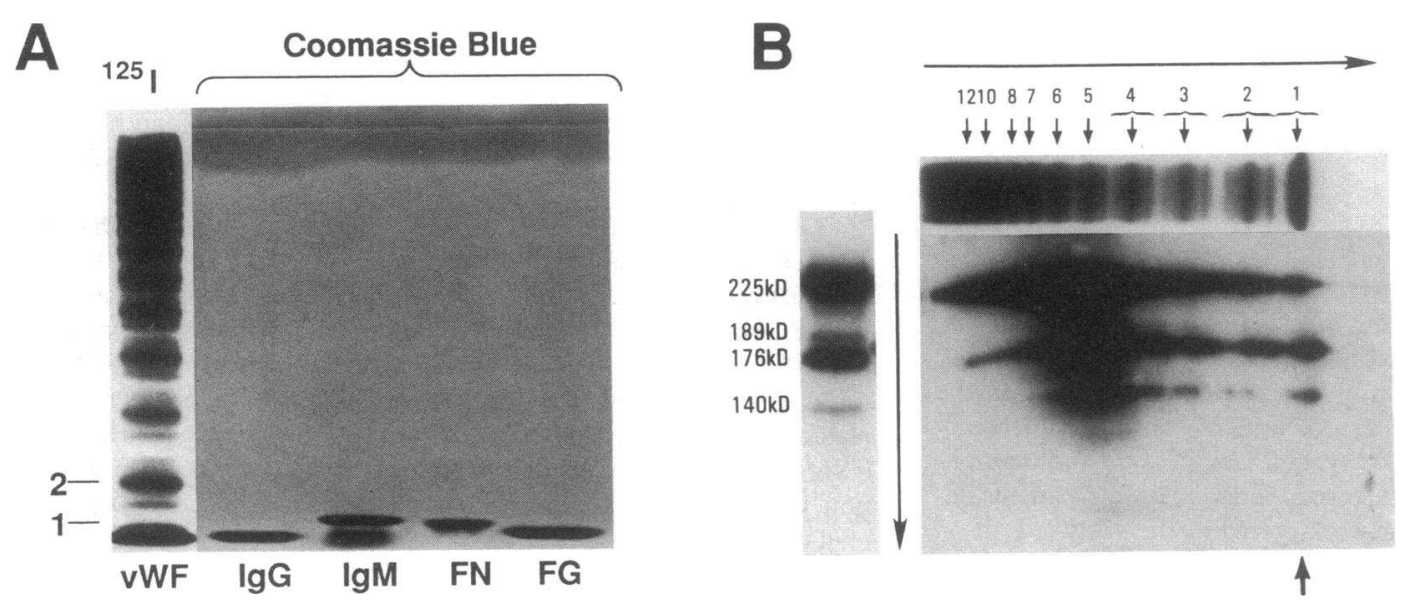

Figure 1. Intermediate resolution one- and twodimensional analysis of immunopurified vWf from normal plasma. $(A)$ Electrophoretic analysis of normal plasma vWf and marker proteins in a nonreduced "short" SDS-agarose gel. The marker proteins are mouse IgG, mouse IgM, human fibronectin $(F N)$, and human fibrinogen (FG) stained with Coomassie Blue, as indicated; vWf multimers are visualized by incubation with ${ }^{125}$ I-labeled rabbit anti-human vWf IgG. (B) Nonreduced/reduced two-dimensional analysis of vWf. The horizontal arrow at the top indicates the direction of migration in the "short" first dimension agarose gel. A lane incubated with ${ }^{125}$ I-labeled rabbit anti-human vWf after the first dimension electrophoresis serves as reference for the position of unreduced vWf multimers (top). Numbered brackets indicate the first four "triplets" resolved in the gel. Small vertical arrows denote the central, more intense band of each triplet (up to twelve distinct bands could be seen in the original autoradiogram). The left hand vertical arrow indicates the direction of migration of the second-dimension electrophoresis. Reduced immunopurified vWf serves as a marker for the second-dimension gel (left hand side). Polypeptides separated in the second dimension are visualized by incubation with a pool of monoclonal antibodies known to react with epitopes in all domains of the vWf subunit. The arrow at the lower right indicates the dye front of the first-dimension gel. The apparent molecular mass of reduced polypeptides is indicated on the left in kD.

ing in molecular mass from $\sim 160(\mathrm{IgG})$ to $900 \mathrm{kD}(\mathrm{IgM})$ were minimally separated in this type of gels, suggesting that the intense band at the dye front could represent several vWf multimers of smaller size not separated from one another (Fig. $1 \mathrm{~A}$ ).

Second-dimension SDS-PAGE analysis after reduction of disulfide bonds demonstrated that the largest $\mathrm{vWf}$ species at the top of the first-dimension gel contain only intact subunit, while multimers of intermediate and smaller size contain also 189-, 176-, and 140-kD subunit fragments (Fig. $1 B$ ). The 140-kD species was particularly evident in multimers of intermediate size; it could not be detected in some of the larger species in which $176-\mathrm{kD}$ fragment was present, and its intensity decreased in smaller multimers (Fig. $1 B$ ). In order to visualize the minor $140-\mathrm{kD}$ species it was necessary to analyze autoradiographs exposed for a prolonged period of time. This caused saturation of the film emulsion laying over the bands containing greater radioactivity, thus explaining the different intensity of corresponding bands in the one- and two-dimension gels shown in Fig. 1. An apparent fragment with mobility intermediate between the 176- and $140-\mathrm{kD}$ species, as well as a fainter one below the $140-\mathrm{kD}$ fragment, could be seen in the second dimension gel in correspondence with multimers of intermediate size. These minor species, not seen in reduced one-dimensional gels (see the left lane in Fig. $1 B$ ), may result from proteolytic cleavage of one or more peptide bonds different from the one identified previously (19); or they may be artifacts due to abnormal disulfide reduction and/or migration during the second-dimension electrophoresis.

High resolution SDS-agarose electrophoresis of normal plasma vWf (Fig. $2 \mathrm{~A}$ ) confirmed a previously (11) described pattern of repeating "pentuplets", rather than the "triplets" seen in intermediate resolution gels, consisting of a more intense central band (band 1) flanked by four less intense satellite bands, two above it (bands $1 \mathrm{~A}$ and $2 \mathrm{~A}$ ) and two below it (bands $1 B$ and $2 B$ ). This terminology, proposed in a previous publication (11), appears to be supported by the symmetry of bands around the central one. The same pattern was clearly visible in at least three sets of five bands of progressively slower mobility, but resolution of individual species became gradually less distinct for the largest multimers towards the top of the first-dimension gel (Fig. 2). The relative mobility of marker proteins demonstrated that high resolution gels allow separation of smaller vWf oligomers with no stacking at the dye front (Fig. 2 $A$ ). Based on the relative position of the IgM marker, it is apparent that the main band in the first distinct "triplet", seen in intermediate resolution gels (identified as band 2 in Fig. $1 \mathrm{~A}$ ), corresponds to main band 2 in the series of $\mathrm{vWf}$ oligomers (Fig. $2 A$ ); and that at least five vWf oligomers migrate at the front of intermediate resolution gels without being separated (the five bands included in the bracket in Fig. $2 A$, corresponding to the unresolved band 1 in Fig. $1 A$ ).

Reduction and second dimension electrophoresis demonstrated the heterogeneous subunit composition of normal plasma vWf multimers (Fig. $2 \mathrm{~B}$ ). This evaluation was facilitated by the concurrent analysis of plasma vWf isolated from patients with Type IIA von Willebrand disease with increased concentration of the two major proteolytic fragments (Fig. 2 $C)$. The distribution of bands in the nonreduced first dimension gels was similar in normal and IIA vWf, but the largest multimers were decreased and satellite bands showed relatively increased intensity in the latter. In accordance with previously reported observations (14), the $189-\mathrm{kD}$ fragment was markedly decreased or not detectable in IIA vWf. A new terminology for the identification of individual oligomers is illustrated schematically in Fig. 3, and is representative of the subunit composition of plasma vWf from 23 normal individuals, five IIA, and four IIB von Willebrand disease patients examined over the course of this study. No differences were noted between normal individuals and patients, except for those specifically described below. In spite of the significant degree of heterogeneity, repeating patterns of subunit composition could be clearly identified in multimers of increasing molecular mass; thus, the structure 


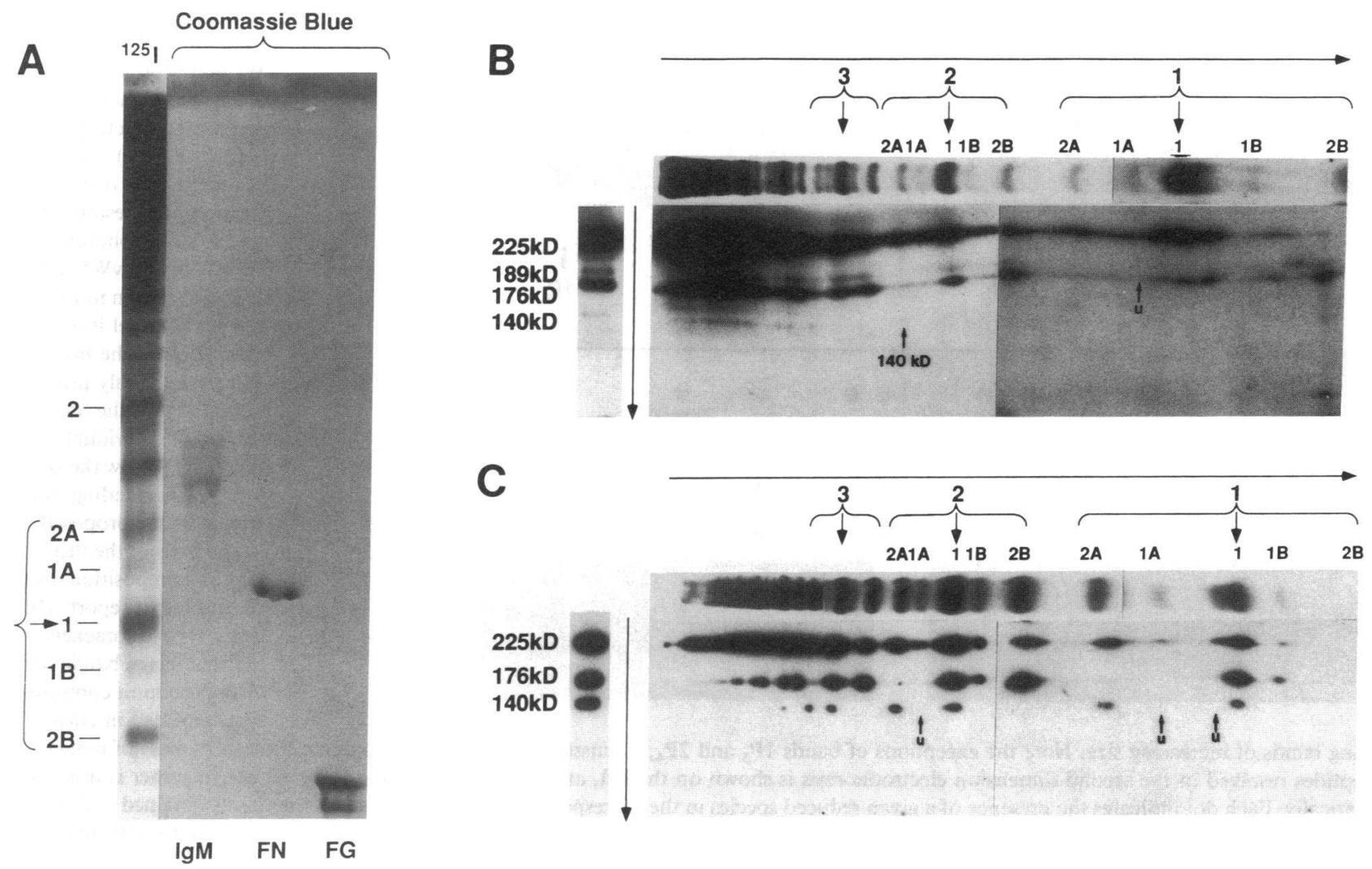

Figure 2. High resolution one- and two-dimensional analysis of vWf immunopurified from normal plasma and from a patient with Type IIA von Willebrand disease. $(A)$ One-dimensional electrophoretic analysis of normal plasma vWf and marker proteins in a nonreduced "long" agarose gel of high resolving power. $(B)$ Analysis of immunopurified normal plasma vWf by high resolution nonreduced/reduced two-dimensional gel electrophoresis. $(C)$ Analysis similar to that shown in $B$, but performed with plasma vWf from a patient with type IIA von Willebrand disease. The experimental details are essentially identical to those described in the legend to Fig. 1, except for the type of agarose gel used in the first dimension. In $B$ and $C$, the horizontal arrow on top of each first dimension gel and the vertical arrow on the left indicate the direction of migration in the first and second dimension, respectively. The three fastest migrating sets of repeating bands are indicated by brackets; each contains five species labeled $2 \mathrm{~A}, 1 \mathrm{~A}, 1,1 \mathrm{~B}$, and $2 \mathrm{~B}$ (from the slowest to fastest migrating band within each set). The letter $U$ indicates the position of bands composed of unproteolyzed mature $\mathrm{vWf}$ subunit. In smaller normal multimers $(B)$, the $140-\mathrm{kD}$ fragment is barely detectable; the position of the smallest species containing an identifiable amount of such subunit fragment is indicated by an arrow. Note that the larger IIA vWf multimers $(C)$ appear to contain less $176-\mathrm{kD}$ fragment than the corresponding normal ones $(B)$; however, the amount of fragment appears to be proportional to the lesser amount of intact subunit, reflecting the marked decrease of larger species in IIA vWf.

of unresolved larger multimers can be reasonably derived by analogy with that of the smaller ones, as outlined in detail below.

The bands previously identified as $1 \mathrm{~A}$ are comprised exclusively of intact subunit. The first (smallest) oligomer in this series has an electrophoretic mobility compatible to its being a dimer, moving just more slowly than fibronectin (molecular mass $445 \mathrm{kD}$; Fig. 2A). These species are identified with the letter $U$, for unproteolyzed, and numbered from the smallest one ( $1 U$ ) in ascending order (Fig. 3). All the bands containing cleaved subunit fragments and migrating faster than $1 \mathrm{U}$ are identified with the letter $P$, for proteolyzed, preceded by the number 1 to indicate that they belong to the first set of oligomers of repeating subunit structure. This notation is completed by a suffix number defining the position in the gel relative to the corresponding unproteolyzed band; accordingly, $1 \mathbf{P}_{1}$ indicates the first proteolyzed band below $1 \mathrm{U}$. The smallest species of this group, $1 \mathrm{P}_{3}$ according to the new terminology (corresponding to a band $2 \mathrm{~B}$ of the previous terminology), appears to be composed exclusively of 176-kD fragment and exhibits a mobility (just slower than that of fibrinogen, molecular mass
$340 \mathrm{kD}$; Fig. $2 A$ ) compatible to its being a dimer of this fragment. The subunit composition of this band can be easily appreciated in Fig. $2 B$ and Fig. 3; its absence in Fig. $2 C$ is caused by excessively long migration of the first dimension gel. Both Fig. $2 B$ and Fig. 3 show a minor species composed only of $189-\mathrm{kD}$ fragment, most likely a homodimer, that is not clearly distinct from bands $1 P_{3}$ and $1 P_{2}$ in the first dimension gel. Fig. $2 \mathrm{~B}$ also suggests the possible existence of a heterodimer composed of one 176-kD and one $189-\mathrm{kD}$ fragment. The 176- and $189-\mathrm{kD}$ dimers are the only two patterns of subunit composition not found in oligomers of greater molecular mass, all containing intact subunit in addition to fragments.

Band $1 \mathrm{P}_{2}$ (1B according to the old terminology) contains intact $225-\mathrm{kD}$ subunit and $176-\mathrm{kD}$ fragment, while band $1 \mathrm{P}_{1}$ (previously designated 1 in each set of repeating "pentuplets") appears to be heterogeneous. This vWf oligomer, predominant in quantity, is not sharply defined in the first dimension gel and appears to be composed of intact subunit as well as variable amounts of 189-, 176-, and 140-kD fragments. Indeed, this band appears as a doublet under ideal resolving conditions (Figs. $2 B, 2 C$, and 3) and may represent different species 


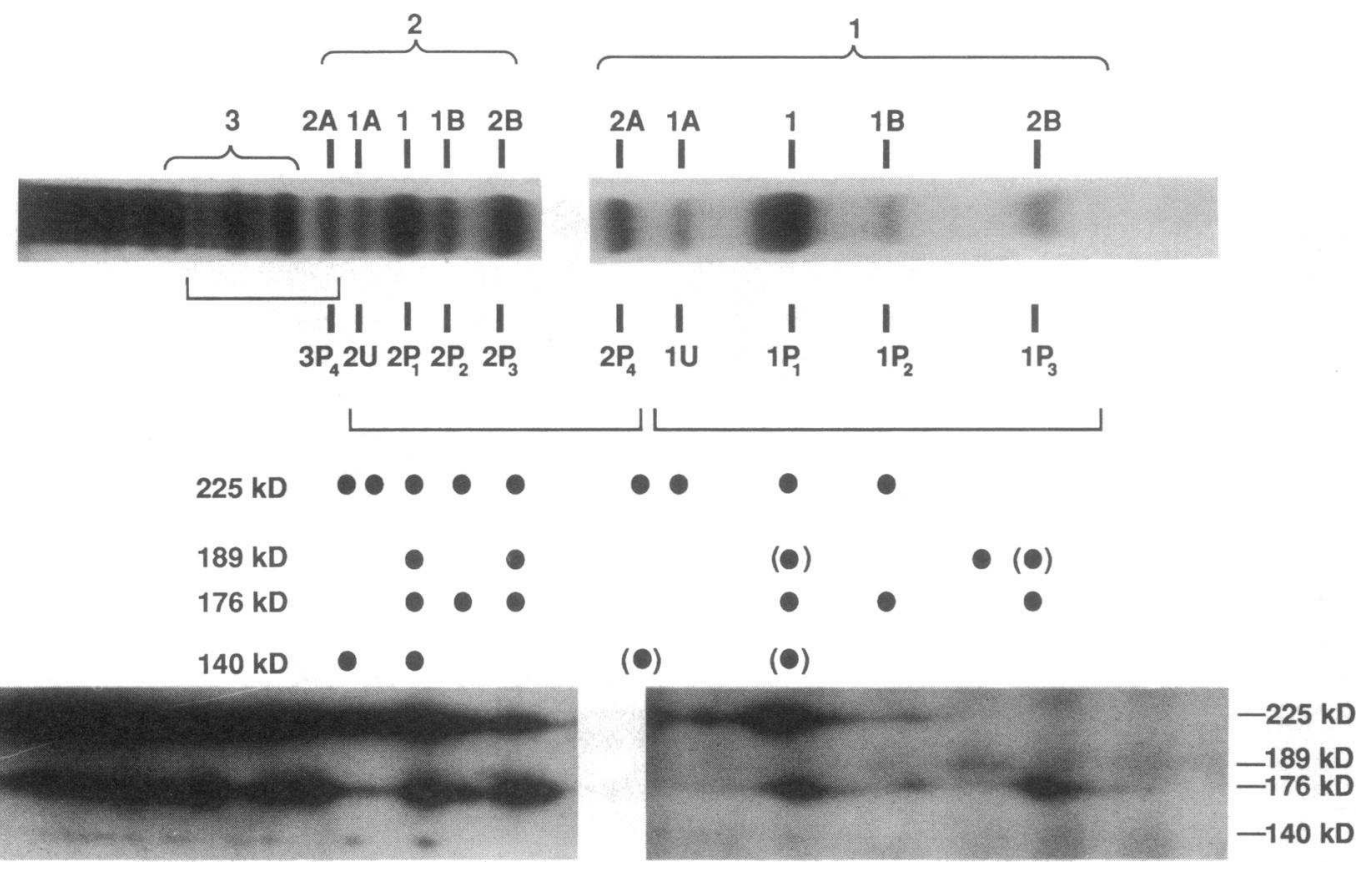

Figure 3. Schematic representation of reduced vWf polypeptides resolved in the second dimension gel electrophoresis (SDS-PAGE). The top gel represents a one-dimension high resolution agarose electrophoretic analysis of IIA vWf, similar to that shown in Fig.

2. Above the gel is a representation of the nomenclature previously proposed (11) for the identification of individual $\mathrm{vWf}$ oligomers. Below the gel is the corresponding new nomenclature proposed on the basis of the distinct subunit composition discussed in this report. Different types of brackets indicate sets of bands with distinct subunit composition, repeated in corre-

sponding bands of increasing size. Note the exceptions of bands $1 \mathbf{P}_{3}$ and $2 \mathrm{P}_{4}$, discussed in Results. The apparent molecular mass of reduced polypeptides resolved in the second dimension electrophoresis is shown on the left, and the subunit composition of each oligomer is indicated schematically. Each dot indicates the presence of a given reduced species in the corresponding oligomer, based on the results obtained with all the samples analyzed during the course of these studies and exemplified in Figs. 2 and 3. Dots in parenthesis indicate subunit fragments that, for a given oligomer, have been seen in some but not all samples.

running in close proximity during SDS-PAGE. The heterogeneity of the main band can be appreciated by a comparison of normal and IIA von Willebrand disease plasma in high resolution agarose gels; this demonstrates a doublet in normal vWf and a predominant band in IIA vWf that corresponds more closely to the lower member of the normal doublet (Fig. 4). With regard to subunit composition, the upper member of the doublet may contain dimers composed only of $225-\mathrm{kD}$ intact subunit, whereas the lower member may contain, in addition, 189-kD fragment, sometimes not seen in IIA vWf, 176-kD fragment, visible in all samples, and 140-kD fragment, particularly evident in some IIA vWf samples (Figs. $2 B, 2 C$, and 3). Thus, the diffuse band $1 P_{1}$ appears to contain some species that, in spite of having identical composition to band $1 U$ (intact subunit) or band $1 P_{2}$ (intact subunit and $176-\mathrm{kD}$ fragment), display different electrophoretic mobility in nonreduced agarose gels. Because all these bands are likely to be dimers, with apparent molecular mass between $\sim 380$ and 480 $\mathrm{kD}$ (see mobility relative to fibronectin and fibrinogen, Fig. 2 $A$ ), it is apparent that factors other than variations in subunit composition must contribute to their different migration (see Discussion).

Multimers of increasing size repeat essentially the subunit composition patterns described so far, with some notable exceptions. Four bands appear between band $2 U$, the second in the series of unproteolyzed oligomers with increasing molecular mass, and band $1 \mathrm{U}$ (Figs. $2 B, 2 C$, and 3). Of these, bands $2 P_{1}$ and $2 P_{2}$ exhibit the same subunit composition as the corresponding bands $1 \mathrm{P}_{1}$ and $1 \mathrm{P}_{2}$, while band $2 \mathrm{P}_{3}$ contains intact subunit and 176-kD fragment, a composition similar to that of bands designated as $P_{2}$; the corresponding band $1 P_{3}$, as dis-

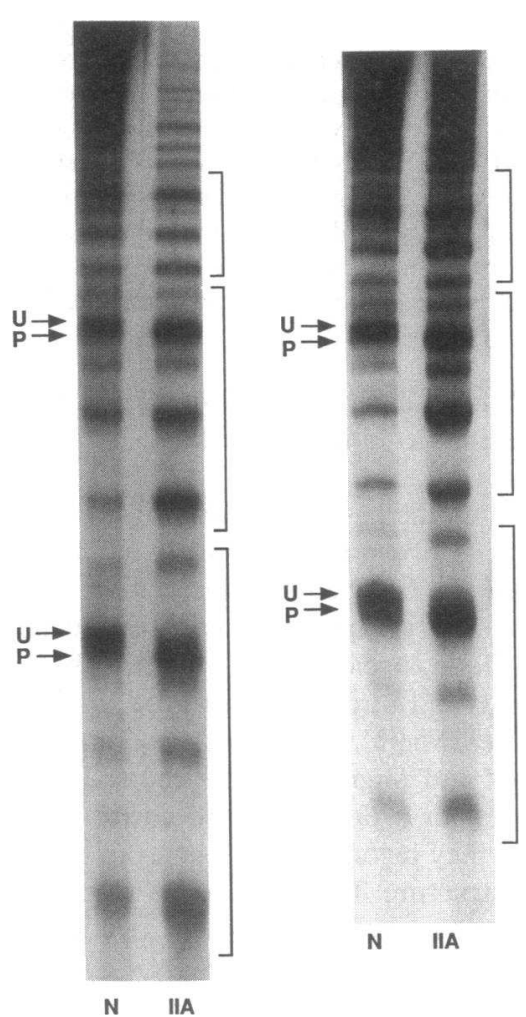

Figure 4. Comparative high resolution agarose gel electrophoresis of immunopurified normal and IIA vWf. This one-dimension analysis was performed as described in the legend to Fig. 2 for comparing the resolution and mobility of main bands in plasma vWf from normal individuals $(N)$ and patients with type IIA von Willebrand disease (IIA). Brackets encompass the bands that, according to the new nomenclature, represent the different patterns of subunit composition repeated in multimers of increasing size. Three such sets of bands are identified; the first at the bottom of the gel is composed of four bands, and all the others of five (see Results). Note that the main (most intense) band in the first two sets of normal vWf multimers is resolved as a doublet; the lower member of the doublet, with slightly increased mobility, is the predominant species in IIA vWf. Such resolution is lost for the species of greater molecular mass. 
cussed above, has no intact subunit. Some species may contain $189-\mathrm{kD}$ fragment instead of $176-\mathrm{kD}$. Band $2 \mathrm{P}_{4}$ (the first in the series of bands corresponding to those designated as $2 \mathrm{~A}$ with the previous terminology) contains intact subunit and $140-\mathrm{kD}$ fragment, the latter more visible in some IIA vWf samples (Figs. $2 \mathrm{~B}, 2 \mathrm{C}$, and 3). Oligomers of increasing molecular mass are likely to have subunit composition corresponding to one of the patterns described for bands $2 \mathrm{U}$ through $2 \mathrm{P}_{4}$, the only difference being an increasing relative number of intact subunits. It should be noted that, even though the fragment of $140 \mathrm{kD}$ is more visible in IIA vWf than in normal, particularly in the smaller oligomers, its presence can be seen upon prolonged exposure of autoradiographs, as shown in Fig. $1 \mathrm{~B}$, and always in a position corresponding to species $\mathrm{P}_{1}$ and $\mathrm{P}_{4}$ (or 1 and $2 \mathrm{~A}$, according to the old terminology). Also to be noted is the fact that the concentration of $176-\mathrm{kD}$ fragment relative to that of intact subunit appears to be increased in $P_{1}$ and $P_{2}$ bands of Type IIA plasma vWf as compared to normal (compare upper and lower panels in Fig. $2 \mathrm{~B}$ ). The different relative content of intact subunit or fragments in corresponding bands from different samples will be discussed below.

Unlike plasma vWf, normal platelet vWf prepared by sonication of washed platelets in the presence of $10 \mathrm{mM}$ EDTA alone, or a combination of $10 \mathrm{mM}$ EDTA, $6 \mathrm{mM} \mathrm{NEM,} 1 \mathrm{mM}$ leupeptin, and $200 \mathrm{KIU}$ aprotinin per $\mathrm{ml}$, was composed only of intact $225-\mathrm{kD}$ subunit in all the multimers, without any evidence of proteolytic fragmentation (shown for the sample prepared with $10 \mathrm{mM}$ EDTA in Fig. 5). Proteolysis was evident when sonication was performed in the absence of proteinase inhibitors (not shown). EDTA alone proved to be as effective as the combination of the four inhibitors in preventing proteolysis.

\section{Discussion}

These results provide a structural explanation for the heterogeneity of circulating vWf multimers. These are originally composed of a varying number of intact $225-\mathrm{kD}$ subunit dimers, but proteolysis occurring as a physiologic event in normal individuals (14) causes the appearance of molecules containing distinct subunit fragments. The two predominant species of 140 and $176 \mathrm{kD}$ originate from a single cleavage of the peptide bond between residues $\mathrm{Tyr}^{842}-\mathrm{Met}^{843}$ of the mature subunit, and represent $v W f$ residues $1-842$ and 843-2050, respectively (19). A third fragment of $189 \mathrm{kD}$, the amino terminal sequence of which has not been possible to determine because of its low abundance $(18,19)$, corresponds essentially to the $176-\mathrm{kD}$ fragment but appears to have a short amino terminal extension overlapping with the carboxyl terminal end of the $140-\mathrm{kD}$ fragment (18). For the purpose of interpreting the subunit structure of $\mathrm{vWf}$ multimers (see below), the 189- and 176-kD species can be considered equivalent.

Because of the disulfide bond distribution in the vWf subunit, cleavage of the peptide bond between residues 842-843 yields two separate species. There are no inter-chain disulfide bonds in the segment of sequence between residues 730-1114, that can be isolated as a single chain tryptic fragment $(27,28)$, and the vWf sequence between residues $730-842$ contains no cysteine (7). Thus, there are no disulfide bonds linking the two subunit fragments generated by cleavage of the $842-843$ bond.

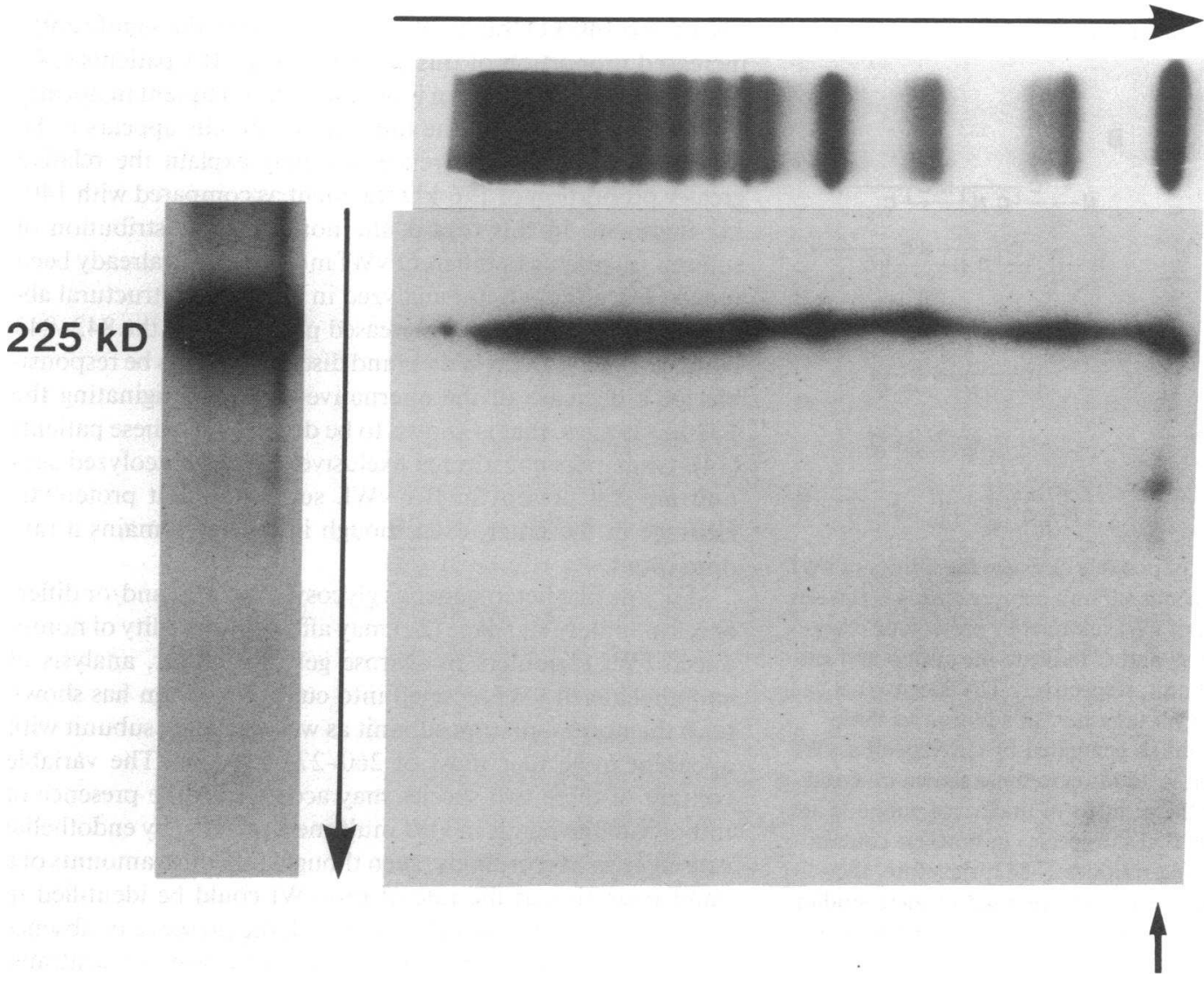

Figure 5. Two-dimensional analysis of affinity-purified normal platelet vWf. An intermediate resolution agarose gel was used in the first dimension to analyze platelet vWf prepared by sonication in the presence of $10 \mathrm{mM}$ EDTA. (See Fig. 1 for experimental details.) Arrows indicate directions of first- and second-dimension electrophoresis. Only unproteolyzed 225-kD subunit can be detected. Minimal amounts of a fragment of smaller molecular mass can be seen at the dye front (small arrow at bottom right). 
Considering that vWf subunits are thought to be assembled tail-to-tail to form dimers and head-to-head to form multimers (29), cleavage of bond 842-843 in any subunit within a vWf molecule yields two separate species of smaller size (Fig. 6). Thus, a single cleavage occurring in the middle of a long oligomer may have profound effects on its size and, because of the known correlation between size and affinity for platelet receptors $(30,31)$, may alter its function in supporting platelet adhesion and aggregation (1).

According to the concepts outlined above, three molecular species can originate from cleavage of the 842-843 peptide bond in dimers of 225-kD intact subunit, namely: (a) 140-kD fragments; $(b)$ 176-kD fragment homodimers; (c) heterodimers of one intact subunit and one 176-kD fragment (Fig. $6 \mathrm{~A}$ ). In some multimers, the $189-\mathrm{kD}$ fragment may replace the $176-\mathrm{kD}$ one. Indeed, all these species have been detected in plasma vWf (Figs. 2 and 3), with the only exception of $140-\mathrm{kD}$ fragments. Regardless of their existence, these species could not be present in the samples analyzed because the monoclonal anti-vWf antibody used for the initial affinity purification recognizes an epitope in the carboxyl terminal region of the subunit and, therefore, cannot interact with molecules containing only the first 842 residues. The same reason may explain why the possible counterpart of the $189-\mathrm{kD}$ fragment, namely one slightly smaller than the $140-\mathrm{kD}$ fragment, has never been identified in $\mathrm{vWf}$ multimers, if the alternative cleavage giving origin to it occurs only in the outermost subunits.

Cleavage of vWf tetramers (Fig. $6 \mathrm{~B}$ ) or larger oligomers (Fig. $6 C$ ) yields several more species with distinct subunit composition, in addition to the ones described above. One species, composed of two or more intact subunits and two $140-\mathrm{kD}$ fragments, but no $176-\mathrm{kD}$ fragments, can derive only from hexamers or larger multimers, but not from tetramers (Fig. 6 $C)$. In general, if an unproteolyzed molecule undergoes one

A

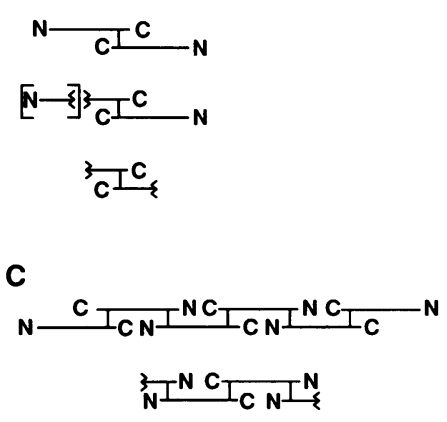

B

$$
\begin{aligned}
& \mathrm{N}-\mathrm{C}_{T_{\mathrm{C} \mathrm{N}}{ }^{\mathrm{N} \mathrm{C}}}{ }_{T_{\mathrm{C}}} \mathrm{N} \\
& {\left[\mathrm{N}-33^{C_{T_{N}}{ }^{N} C_{T_{C}}}\right.} \\
& \mathrm{N} \stackrel{\mathrm{C}_{I_{C}}}{{ }_{N} I^{N C}}{ }_{I_{C}} \mathrm{~N} \\
& { }_{3}^{\mathrm{C}_{\Gamma_{\mathrm{CN}}{ }^{\mathrm{N} \mathrm{C}}} \bar{T}_{\mathrm{C}}} \\
& { }_{\mathrm{N}}^{2} \stackrel{N \mathrm{C}_{T}}{\mathrm{C}} \\
& \stackrel{C_{I_{C}}}{\rightleftarrows}\left[\frac{3}{N}\right]
\end{aligned}
$$

Figure 6. Schematic illustration of possible cleavage modalities of $\mathrm{vWf}$ oligomers resulting in heterogeneous subunit composition. $(A)$ Species that can originate from a dimer of $\mathrm{vWf}$ subunits by proteolytic cleavage at the $\mathrm{Tyr}^{842}-\mathrm{Met}^{843}$ bond. $N$ and $C$ indicate the amino and carboxyl terminal end of each subunit, respectively. $(B)$ Species that can be generated by cleavage of a vWf tetramer, in addition to those shown in $A$. $(C)$ Species that can be generated by cleavage of a $\mathrm{vWf}$ hexamer or any larger oligomer, in addition to those shown in $A$ and $B$. Species that differ only for the number of uncleaved subunits are not specifically identified. Note that the species in brackets contain only vWf sequence encompassing residues $1-842$; therefore, they could not react with the monoclonal antibody used in these studies for affinity purification of $\mathrm{vWf}$ and could not be present in the samples analyzed. single cleavage in any subunit, except for the two at the extreme ends of the oligomer, the two resulting molecules of smaller mass will be composed of a variable number of intact subunits as well as one $140-\mathrm{kD}$ or one $176-\mathrm{kD}$ fragment. If, on the other hand, a single cleavage occurs in one or both of the two subunits at the extreme ends of a polymer, one segment of sequence (140-kD fragment) will be detached from the molecule and the remaining species will contain intact subunits and one or two $176-\mathrm{kD}$ fragments. If more than one inner cleavage occurs, the resulting new molecules may contain a variable number of intact subunits (one or more) as well as one 176-kD and one $140-\mathrm{kD}$ fragment. Thus, only proteolytic degradation of inner subunits can generate multimers containing $140-\mathrm{kD}$ fragment, and molecules containing both $176-\mathrm{kD}$ and $140-\mathrm{kD}$ fragments must have undergone at least two cleavages in distinct subunits (Fig. 6). Unlike unproteolyzed molecules, containing only an even number of subunits, proteolyzed molecules can contain an odd subunit number.

This proposed model of proteolysis, accounting for a maximum of two proteolyzed subunits in each $\mathrm{vWf}$ oligomer (one at each end), explains the experimental observation that the proportion of fragments relative to intact subunit decreases with increasing multimer size, because the number of intact subunits increases progressively in the latter. It also accounts for the fact that larger multimers contain 176-kD fragment in larger proportion than $140-\mathrm{kD}$ fragment, because these species are likely to have been cleaved only at the extreme ends, with minimal reduction in molecular mass. In Type IIA von Willebrand disease, both the increased concentration of subunit fragments and the lack of larger multimers may result from enhanced proteolysis of inner subunits in molecules originally synthesized with normal size $(14,19,32-34)$. As discussed above, this type of cleavage generates multimers containing one or two $140-\mathrm{kD}$ fragments, thus explaining the significantly increased proportion of this species in Type IIA patients (14). This pattern of degradation is obviously less frequent in normal vWf, where cleavage of the outermost subunits appears to be the most common occurrence and may explain the relative greater proportion of $176-\mathrm{kD}$ fragment as compared with 140 $\mathrm{kD}$ fragment. In this regard, the nonuniform distribution of subunit fragments in different $\mathrm{vWf}$ multimers has already been noted (35), although not analyzed in detail. The structural abnormality responsible for increased proteolysis of the 842-843 bond in Type IIA von Willebrand disease may also be responsible for a decrease in the alternative cleavage originating the 189-kD species, that is known to be decreased in these patients (14). Oligomers constituted exclusively of unproteolyzed subunit are still present in IIA vWf, suggesting that proteolytic cleavage in the latter, even though increased, remains a random event.

Factors like heterogeneous glycosylation (27) and/or differences in molecular shape (29) may affect the mobility of nonreduced vWf oligomers in agarose gels. Moreover, analysis of endothelial cell vWf secreted into culture medium has shown both the normal mature subunit as well as a large subunit with apparent molecular mass of 260-275 kD (36). The variable content of these two species may account for the presence of minor satellite bands in vWf multimers secreted by endothelial cells in vitro. Accordingly, even though only trace amounts of a band migrating at the rate of pro-vWf could be identified in some of the plasma samples analyzed, the presence or absence of small quantities of pro-vWf and its uncleaved but posttrans- 
lationally modified form may account for some of the heterogeneity seen in the size distribution of circulating $\mathrm{vWf}$ molecules. Thus, a combination of various causes may explain the observed lack of sharp definition of $\mathrm{vWf}$ bands in nonreduced agarose gels, as well as the fact that multimers of similar subunit composition migrate differently or, conversely, multimers with apparently different subunit composition migrate in close proximity.

It is not surprising, therefore, that corresponding multimer bands in normal and IIA vWf may contain different relative proportions of intact subunit and fragments because, in fact, they represent species with subtle structural differences. For example, band $1 P_{1}$, that can be resolved into a doublet, contains more $225-\mathrm{kD}$ subunit than $176-\mathrm{kD}$ fragment in normal, and contains $140-\mathrm{kD}$ fragment in IIA vWf that is barely visible in normal. This may be explained assuming that band $1 P_{1}$ represents the following distinct species migrating in close proximity: homodimers of intact subunits; heterodimers of $225-\mathrm{kD}$ subunit and 176-kD fragment; and, predominantly in IIA vWf, molecules composed of one $225-\mathrm{kD}$ subunit, one $176-\mathrm{kD}$, and one $140-\mathrm{kD}$ fragment, derived from two internal cleavages in tetramers or any larger multimer (Fig. 6). In a similar manner, band $1 \mathrm{P}_{4}$ appears to contain mainly intact subunit in normal vWf but also $140-\mathrm{kD}$ fragment in IIA vWf. This may be explained if the band represents intact dimers as well as molecules composed by two $225-\mathrm{kD}$ subunits and one $140-\mathrm{kD}$ fragment (Fig. $6 \mathrm{~B}$ ). The same subunit composition patterns would be repeated in larger multimers, with the only addition of more intact subunits. Definitive demonstration of the validity of these concepts will require improved separation of $\mathrm{vWf}$ oligomers allowing accurate quantitative analysis of the corresponding subunit composition.

In conclusion, these studies define a prevalent mode of posttranslational modification of the constituent subunit of circulating $\mathrm{vWf}$. Whether or not proteolytic cleavage contributes to the conversion of extra large (or "unusually large") vWf molecules secreted by endothelial cells (37) to the smaller forms normally present in plasma remains to be established. Indeed, the concepts and methodology established here can aid in the study of normal vWf processing as well as of congenital and acquired structural alterations of $\mathrm{vWf}$ multimers.

\section{Acknowledgments}

The authors wish to thank James R. Roberts for his help in the preparation of monoclonal antibodies, Marina Hoffman for editorial assistance, and Eileen Bristow for secretarial assistance.

This work was supported by grants HL-15491 and HL-31950 from the National Institutes of Health. This is publication No. 6407-MEM/ CVB from the Research Institute of Scripps Clinic.

\section{References}

1. Ruggeri, Z. M., and T. S. Zimmerman. 1987. von Willebrand factor and von Willebrand disease. Blood. 70:895-904.

2. Bonthron, D., E. C. Orr, L. M. Mitsock, D. Ginsburg, R. I. Handin, and S. H. Orkin. 1986. Nucleotide sequence of pre-pro-von Willebrand factor cDNA. Nucleic Acids Res. 14:7125-7127.

3. Lynch, D. C., R. Williams, T. S. Zimmerman, E. P. Kirby, and D. M. Livingston. 1983. Biosynthesis of the subunits of factor VIIIR by bovine aortic endothelial cells. Proc. Natl. Acad. Sci. USA. 80:2738-2742.

4. Wagner, D. D., and V. J. Marder. 1983. Biosynthesis of von Willebrand protein by human endothelial cells: identification of a large precursor polypeptide chain. J. Biol. Chem. 258:2065-2067.

5. Wagner, D. D., and V. J. Marder. 1984. Biosynthesis of von Willebrand protein by human endothelial cells: processing steps and their intracellular localization. J. Cell Biol. 99:2123-2130.

6. Wagner, D. D., S. O. Lawrence, B. M. Ohlsson-Wilhelm, P. J. Fay, and V. J. Marder. 1987. Topology and order of formation of interchain disulfide bonds in von Willebrand factor. Blood. 69:27-32.

7. Titani, K., S. Kumar, K. Takio, L. H. Ericsson, R. D. Wade, K. Ashida K. A. Walsh, M. W. Chopek, J. E. Sadler, and K. Fujikawa. 1986. Amino acid sequence of human von Willebrand factor. Biochemistry. 25:3171-3184.

8. Montgomery, R. R., and T. S. Zimmerman. 1978. von Willebrand's disease antigen II. A new plasma and platelet antigen deficient in severe von Willebrand's disease. J. Clin. Invest. 61:1498-1507.

9. Fay, P. J., Y. Kawai, D. D. Wagner, D. Ginsburg, D. Bonthron, B. M. Ohlsson-Wilhelm, S. I. Chavin, G. N. Abraham, R. I. Handin, S. H. Orkin, R. R. Montgomery, and V. J. Marder. 1986. Propolypeptide of von Willebrand factor circulates in blood and is identical to von Willebrand antigen II. Science (Wash. DC). 232:995-998

10. Ruggeri, Z. M., and T. S. Zimmerman. 1981. The complex multimeric composition of Factor VIII/von Willebrand factor. Blood. 57:1140-1143.

11. Ciavarella, G., N. Ciavarella, S. Antoncecchi, D. De Mattia, P. Ranieri, J. Dent, T. S. Zimmerman, and Z. M. Ruggeri. 1985. High resolution analysis of von Willebrand factor multimeric composition defines a new variant of Type I von Willebrand disease with aberrant structure but presence of all size multimers (Type IC). Blood. 66:1423-1429.

12. Ruggeri, Z. M., I. M. Nilsson, R. Lombardi, L. Holmberg, and T. S. Zimmerman. 1982. Aberrant multimeric structure of von Willebrand factor in a new variant of von Willebrand's disease (Type IIC). J. Clin. Invest. 70:11241127.

13. Kinoshita, S., J. Harrison, J. Lazerson, and C. F. Abildgaard. 1984. A new variant of dominant type II von Willebrand's disease with aberrant multimeric pattern of factor VIII-related antigen (Type IID). Blood. 63:1369-1371.

14. Zimmerman, T. S., J. A. Dent, Z. M. Ruggeri, and L. H. Nannini. 1986. Subunit composition of plasma von Willebrand factor. Cleavage is present in normal individuals, increased in IIA and IIB von Willebrand disease, but minimal in variants with aberrant structure of individual oligomers (Types IIC, IID and IIE). J. Clin. Invest. 77:947-951.

15. Mannucci, P. M., R. Lombardi, A. B. Federici, J. A. Dent, T. S. Zimmerman, and Z. M. Ruggeri. 1986. A new variant of Type II von Willebrand disease with aberrant multimeric structure of plasma but not platelet von Willebrand Factor (Type IIF). Blood. 68:269-274.

16. Gralnick, H. R., S. B. Williams, L. P. McKeown, P. Maisonneuve, C. Jenneau, and Y. Sultan. A variant of Type II von Willebrand disease with an abnormal triplet structure and discordant effects of protease inhibitors on plasma and platelet von Willebrand factor structure. Am. J. Hematol. 24:259-266.

17. Batlle, J., M. F. Lopez Fernandez, J. Lasierra, A. Fernandez Villamor, C. Lopez Berges, A. Lopez Borrasca, Z. M. Ruggeri, and T. S. Zimmerman. 1986. von Willebrand's disease Type IIC with different abnormalities of von Willebrand factor in the same sibship. Am. J. Hematol. 21:177-188.

18. Berkowitz, S. D., J. A. Dent, J. R. Roberts, Y. Fujimura, E. F. Plow, K. Titani, Z. M. Ruggeri, and T. S. Zimmerman. 1987. Epitope mapping of the von Willebrand factor subunit distinguishes fragments present in normal and Type IIA von Willebrand disease from those generated by plasmin. J. Clin. Invest. 79:524-531.

19. Dent, J. A., S. D. Berkowitz, J. Ware, C. K. Kasper, and Z. M. Ruggeri. 1990. Identification of a cleavage site directing the immunochemical detection of molecular abnormalities in type IIA von Willebrand factor. Proc. Natl. Acad. Sci. USA. 87:6306-6310.

20. Liu, F. T., J. W. Bohn, E. L. Terry, H. Yamamoto, C. A. Molinaro, L. A. Sherman, N. R. Klinman, and D. H. Katz. 1980. Monoclonal anti-dinitrophenylspecific murine IgE antibody: preparation, isolation and characterization. $J$. Im munol. 124:2728-2737.

21. Fulcher, C. A., and T. S. Zimmerman. 1982. Characterization of the human Factor VIII procoagulant protein with a heterologous precipitating antibody. Proc. Natl. Acad. Sci. USA. 79:1648-1652.

22. Ey, P. L., S. J. Prowse, and C. R. Jenkin. 1978. Isolation of pure IgG1, IgG2a and IgG2b immunoglobulins from mouse serum using protein A-Sepharose. Immunochemistry. 15:429-436.

23. Parham, P., M. J. Androlewicz, F. M. Brodsky, N. J. Holmes, and J. P. Ways. 1982. Monoclonal antibodies: Purification, fragmentation and application to structural and functional studies of class I MHC antigens. J. Immunol. Methods. 53:133-173.

24. Kazal, L. A., S. Amsel, O. P. Miller, and L. M. Tocantins. 1963. The preparation and some properties of fibrinogen precipitated from human plasma by glycine. Proc. Soc. Exp. Biol. Med. 113:989-994.

25. Engvall, E., and E. Ruoslahti. 1977. Binding of soluble form of fibroblast surface protein, fibronectin, to collagen. Int. J. Cancer. 20:1-5.

26. Laemmli, U. K. 1970. Cleavage of structural proteins during the assembly of the head of bacteriophage T4. Nature (Lond.). 227:680-685.

27. Fujimura, Y., K. Titani, L. Z. Holland, S. R. Russell, J. R. Roberts, J. H. 
Elder, Z. M. Ruggeri, and T. S. Zimmerman. 1986. von Willebrand factor. A reduced and alkylated $52 / 48 \mathrm{kDa}$ fragment beginning at amino acid residue 449 contains the domain interacting with platelet glycoprotein Ib. J. Biol. Chem. 261:381-385.

28. Pareti, F. I., K. Niiya, J. M. McPherson, and Z. M. Ruggeri. 1987. Isolation and characterization of two domains of human von Willebrand factor that interact with fibrillar collagen Types I and III. J. Biol. Chem. 262:13835-13841.

29. Fowler, W. E., L. J. Fretto, K. K. Hamilton, H. P. Erickson, and P. A. McKee. 1985. Substructure of human von Willebrand factor. J. Clin. Invest. 76:1491-1500.

30. Federici, A. B., R. Bader, S. Pagani, M. L. Colibretti, L. De Marco, and P. M. Mannucci. 1989. Binding of von Willebrand factor (vWf) to glycoproteins (GP) Ib and IIb-IIla complex: affinity is related to multimeric size. Br. J. Haematol. 73:93-99.

31. De Marco, L., M. Mazzucato, D. De Roia, A. Casonato, A. B. Federici, A. Girolami, and Z. M. Ruggeri. 1990. Distinct abnormalities in the interaction of purified IIA and IIB von Willebrand factor with the two platelet binding sites GP Ib-IX and GP IIb-IIIa. J. Clin. Invest. 86:785-792.

32. Ginsburg, D., B. A. Konkle, J. C. Gill, R. R. Montgomery, P. L. Bockenstedt, T. A. Johnson, and A. Y. Yang. 1989. Molecular basis of human von
Willebrand disease: analysis of platelet von Willebrand factor mRNA. Proc. Natl. Acad. Sci. USA. 86:3723-3727.

33. Gralnick, H. R, S. B. Williams, L. P. McKeown, P. Maisonneuve, C. Jenneau, Y. Sultan, and M. E. Rick. 1985. In vitro correction of the abnormal multimeric structure of von Willebrand factor in type IIa von Willebrand's disease. Proc. Natl. Acad. Sci. USA. 82:5968-5972.

34. Batlle, J., M. F. Lopez Fernandez, M. Campos, B. Justica, C. Berges, J. L Navarro, J. M. Diaz Cremades, C. K. Kasper, J. A. Dent, Z. M. Ruggeri, and T. S. Zimmerman. 1986. The heterogeneity of Type IIA von Willebrand's disease: studies with protease inhibitors. Blood. 68:1207-1212.

35. Chopek, M. W., J.-P. Girma, K. Fujikawa, E. W. Davie, and K. Titani. 1986. Human von Willebrand factor: a multivalent protein composed of identical subunits. Biochemistry. 25:3146-3155.

36. Lynch, D. C., T. S. Zimmerman, E. H. Ling, and P. J. Browning. 1986. An explanation for minor multimer species in endothelial cell-synthesized von Willebrand factor. J. Clin. Invest. 77:2048-2051.

37. Moake, J. L., C. K. Rudy, J. H. Troll, M. J. Weinstein, N. M. Colannino, J. Azacar, R. H. Seder, S. L. Hong, and D. Deykin. 1982. Unusually large plasma factor VIII: von Willebrand factor multimers in chronic relapsing thrombotic thrombocytopenic purpura. N. Engl. J. Med. 307:1432-1435. 